\title{
Infecção pelo HTLV-1/2 em gestantes brasileiras
}

\author{
$H T L V-1 / 2$ infection in Brazilian pregnant women \\ Infección por el HTLV-1/2 en gestantes brasileras
}

\author{
Danielle B. S. Barmpas, Denise L. M. Monteiro, 'Stella R. Taquette, Alexandre J. B. Trajano, \\ Roberta M. Raupp, Fátima R. D. Miranda, Nadia C. P. Rodrigues
}

\begin{abstract}
Resumo
HTLV-1/2 são retrovírus com três vias de transmissão: sexual, hematogênica e vertical (TV). Em áreas endêmicas, a TV tem o papel principal na cadeia de transmissão, principalmente pelo aleitamento materno. A infecção é perene e $90 \%$ dos pacientes são portadores assintomáticos, porém existe associação com uma gama de doenças inflamatórias, de dermatites inespecíficas até patologias graves e de prognóstico reservado, como a leucemia/ linfoma de células T do adulto (LTA) e a mielopatia associada ao HTLV-1, ou paraparesia espástica tropical (MAH). Até hoje não existem formas de prevenção ou tratamento eficaz para a infecção por HTLV-1/2 nem para as doenças decorrentes da mesma. Os dados sobre a infecção por HTLV-1/2 em gestantes brasileiras ainda são escassos e isolados, pois a maioria dos artigos estuda doadores de sangue, a infecção não tem notificação compulsória e o rastreio não faz parte da rotina pré-natal preconizada pelo Ministério da Saúde. O objetivo deste trabalho é reunir o conhecimento disponível sobre o tema na literatura internacional. Ainda não há estudos publicados sobre a prevalência da infecção em nosso estado, mas o projeto "Estudo da prevalência e da transmissão vertical de HIV, HTLV e Sífilis em gestantes da Região Metropolitana do Rio de Janeiro", de professores da UERJ com financiamento da FAPERJ, identificou prevalência de 0,66\% em parturientes na região metropolitana do Rio de Janeiro. Com base neste resultado, foi proposta a inclusão do exame para o HTLV na rotina pré-natal do HUPE/ UERJ.O melhor conhecimento sobre a prevalência de HTLV-1/2 em gestantes nas diferentes regiões brasileiras é fundamental para orientar a elaboração de políticas educacionais, sociais e de saúde pública que possam conscientizar a população sobre a doença e reduzir as taxas de transmissão vertical.
\end{abstract}

Descritores: HTLV; Doenças sexualmente transmissíveis; Prevalência; Gravidez; Transmissão vertical de doença infecciosa.

\begin{abstract}
HTLV-1/2 are retroviruses with three modes of transmission: sexual, blood borne and from mother to child / vertical (VT). In endemic areas VT plays the major role in the chain of transmission, mainly by breastfeeding. Infection is lifelong and $90 \%$ of the patients are asymptomatic carriers. However, there are associations to a range of inflammatory conditions, from nonspecific dermatitis to severe diseases with poor prognosis such as adult T-cell leukemia/lymphoma (ATL) and HTLV-1 associated myelopathy/tropical spastic paraparesis (HAM/ TSP). So far there is no efficient prevention or treatment of HTLV-1/2 infection or the diseases caused by it. Data on HTLV-1/2 on Brazilian pregnant women is still scarce and isolated because most articles study blood donors, the infection is not notifiable and screening is not part of the antenatal routine recommended by the Ministry of Health. The goal of the present work is to gather the knowledge available on the subject in international literature. There are still no published studies about the prevalence of HTLV-1/2 infection in pregnant women in our State, however the project "Study of the prevalence and vertical transmission of HIV, HTLV and SYPHILIS in pregnant women on the metropolitan area of Rio de Janeiro" carried out by UERJ professors and funded by FAPERJ, identified a prevalence of $0.66 \%$ in women admitted for delivery on the metropolitan area of Rio de Janeiro. Based on this result, the inclusion of HTLV screening in the routine antenatal care of HUPE/UERJ was proposed. Better knowledge about the prevalence of HTLV-1/2 in pregnant women among the diverse Brazilian regions is crucial to guide the development of educational, social and public health policies to increase awareness about the condition and reduce mother to child transmission rates.
\end{abstract}


Keywords: HTLV; Sexually transmitted diseases; Prevalence; Pregnancy; Infectious disease transmission vertical.

\section{Resumen}

HTLV-1/2 son retrovirus con tres vías de transmisión: sexual, hematógena y vertical (TV). En áreas endémicas, la TV tiene el papel principal en la cadena de transmisión, principalmente por el amamantamiento materno. La infección es perenne y el $90 \%$ de los pacientes son portadores asintomáticos, sin embargo existe asociación con una gama de enfermedades inflamatorias, de dermatitis inespecíficas hasta patologías graves y de pronóstico reservado, como la leucemia/linfoma de células T del adulto (LTA) y la mielopatía asociada al HTLV-1, o paraparesia espástica tropical (MAH). Hasta hoy no existen formas de prevención o tratamiento eficaz para la infección por HTLV-1/2 ni para las enfermedades derivadas de la misma. Los datos sobre la infección por HTLV-1/2 en gestantes brasileras todavía son escasos y aislados, pues la mayoría de los artículos estudia donadores de sangre, la infección no tiene notificación establecida y la detección no hace parte de la rutina prenatal preconizada por el Ministerio de Salud. El objetivo de este trabajo es reunir el conocimiento disponible sobre el tema en la literatura internacional. Todavía no hay estudios publicados sobre la prevalencia de la infección en nuestro estado, pero el proyecto "Estudio de la prevalencia y de la transmisión vertical de HIV, HTLV y Sifilis en gestantes de la región metropolitana de Rio de Janeiro”, de profesores de la UERJ con financiamiento de la FAPERJ, identificó prevalencia del $0,66 \%$ en parturientas en la región metropolitana de Rio de Janeiro. Con base en este resultado fue propuesta la inclusión del examen para el HTLV en la rutina prenatal del HUPE/UERJ.Un mejor conocimiento sobre la prevalencia de HTLV-1/2 en gestantes en las diferentes regiones brasileras es fundamental para orientar la elaboración de políticas educacionales, sociales y de salud pública que puedan concientizar a la población sobre la enfermedad y reducir las tasas de transmisión vertical.

Palabras clave: HTLV; enfermedades sexualmente transmisibles; prevalencia; embarazo; transmisión vertical de enfermedad infecciosa.

\section{Introdução}

Os vírus HTLV-1 e 2 foram os primeiros retrovírus humanos identificados na década de 1980, e apresentam forte tropismo por linfócitos-T e transmissão intercelular obrigatória. A infecção causada por eles possui distribuição mundial, estimando-se entre 15 e 20 milhões de pessoas infectadas. $^{1,2}$

A prevalência varia de acordo com a área geográfica, composições sociodemográfica e étnica da população, além de grupos de risco específicos, como usuários de drogas injetáveis, profissionais do sexo e portadores de HIV. O HTLV-1 é endêmico na África Ocidental, sudoeste do Japão e áreas da América Latina; a soroprevalência é maior no sexo feminino e aumenta com a idade. Sua epidemiologia é peculiar em virtude da distribuição de polos endêmicos adjacentes a áreas de baixa ou média prevalência. O HTLV-2 por sua vez está associado a populações "nativas" das Américas e ao uso de drogas injetáveis., ${ }^{1,2}$

A maioria dos infectados (90\%) permanece assintomática, mas é reservatório do vírus e sustenta a cadeia de transmissão. Por outro lado, até 7\% dos portadores desenvolvem manifestações clínicas graves e com prognóstico reservado como leucemia/linfoma de células $\mathrm{T}$ do adulto (ATLL) e mielopatia associada ao HTLV-1/paraparesia espástica tropical (HAM/TSP). A infecção também pode causar uma série de doenças inflamatórias, como uveíte, dermatite e doenças reumatológicas, além de poder cursar com imunodepressão subclínica e patologias oportunistas como estrongiloidíase, hanseníase, escabiose crostosa e tuberculose. O HTLV-2 está associado à leucemia de células pilosas, à eritrodermatite, a polineuropatias e, em coinfectados com HIV, a infecções bacterianas e micoses fungoides. Em virtude de seu caráter silencioso, a infecção e suas vias de transmissão permanecem relativamente desconhecidas até mesmo pelos profissionais de saúde. ${ }^{2}$

As publicações sobre HTLV-1/2 em gestantes brasileiras ainda são escassas, pois a maioria dos estudos aborda a infecção em doadores de sangue. Adicionalmente, não há dados oficiais sobre o tema, já que a infecção não tem notificação compulsória, e o rastreio não faz parte da rotina pré-natal preconizada pelo Ministério da Saúde. Com o objetivo de reunir o conhecimento disponível na literatura, foi realizada revisão sistematizada que identificou os estados brasileiros em que há estudos sobre a prevalência dos vírus em gestantes. Os resultados obtidos foram resumidos e comparados com os dados de prevalência em doadores de sangue. 


\section{Transmissão}

Há três principais vias de transmissão do HTLV-1/2: contato sexual, contato com sangue ou secreções (transfusão de sangue ou hemoderivados celulares, compartilhamento de seringas no uso de drogas injetáveis, em transplantes, exposição percutânea a sangue ou secreções biológicas em profissionais de saúde) e por transmissão vertical (TV). A TV ocorre em cerca de $20 \%$ dos filhos de mães infectadas, sendo sua principal via o aleitamento materno prolongado, ${ }^{3}$ devido à presença de linfócitos infectados no leite. A eficiência da TV também está associada a títulos elevados de anticorpos e alta carga pró-viral materna. ${ }^{4-6} \mathrm{~A}$ transmissão intrauterina ou perinatal não tem impacto epidemiológico significativo; ${ }^{7,8}$ no entanto, sabe-se que 2,5 a $5 \%$ dos filhos de mulheres infectadas serão soroconvertidos, mesmo na ausência de aleitamento materno. ${ }^{3,8} \mathrm{~A}$ infecção intrauterina foi demonstrada através da comprovação histopatológica de infecção placentária em gestantes portadoras de HTLV-1 e da identificação de sequências do vírus em células mononucleares fetais obtidas no sangue do cordão umbilical. ${ }^{9-11}$

\section{Diagnóstico laboratorial}

O diagnóstico laboratorial é realizado por meio de testes de triagem pelas técnicas de imunoensaio ou testes de aglutinação. Os testes de imunoensaio (EIA/ELISA e CMIA) fazem o rastreio para ambos os vírus, enquanto que os de aglutinação detectam apenas o HTLV-1. No Brasil, predominam os testes de imunoensaio devido à presença de ambos os vírus na população. Os testes confirmatórios são capazes de discriminar entre HTLV-1 e HTLV-2. Os principais são Western blot, imunofluorescência indireta (IFA) e radioimunoprecipitação (RIPA). Em casos de resultados

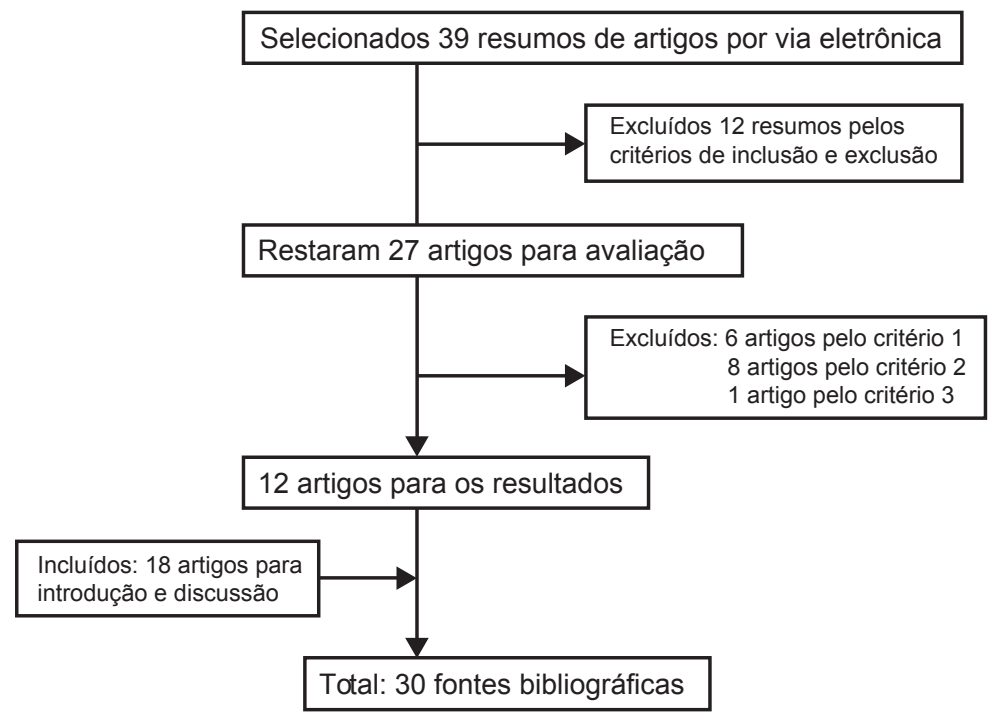

Figura 1. Fluxograma de busca de referências.

Quadro 1. Critérios para o diagnóstico de DMG da International Association of Diabetes and Pregnancy Study Groups -2010.

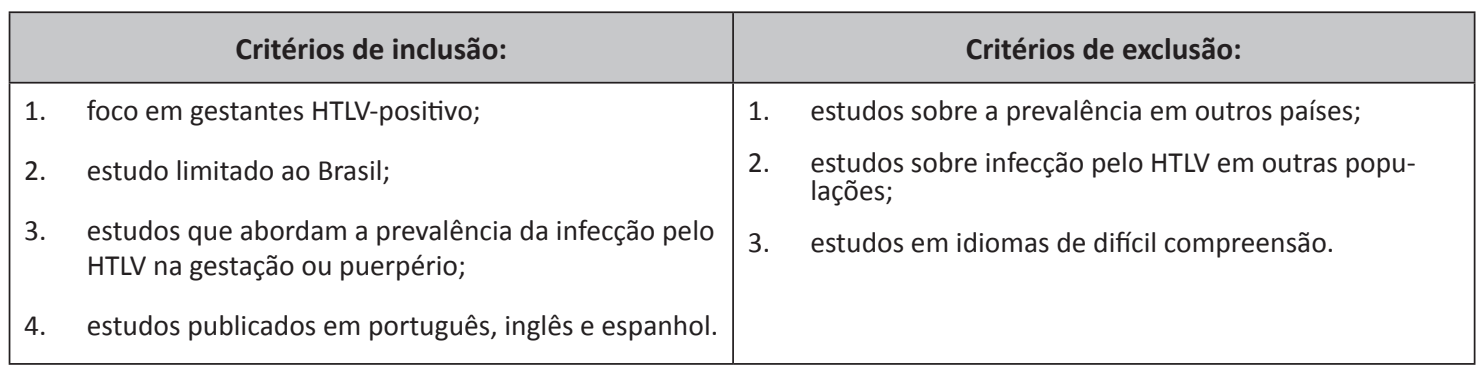


indeterminados nos exames confirmatórios iniciais podem ser utilizados testes confirmatórios moleculares qualitativos ou quantitativos de reação em cadeia da polimerase (PCR).

\section{Método}

Foi realizada ampla pesquisa na literatura médica procurando identificar e extrair informações da literatura nacional e internacional. Esta revisão sistematizada utilizou como bases de dados: Medline (por meio do PubMed), Scielo, LILACS e Google Acadêmico. Para identificação de publicações no Medline (por meio do Pubmed) foi utilizada a seguinte estratégia de busca: ("HTLV-1 Infections"[Mesh]) AND "HTLV-2 Infections"[Mesh]) AND "Pregnancy" [Mesh]) AND "Prevalence"[Mesh], tendo sido encontrados 20 artigos. Para pesquisa realizada nas demais bases foram utilizados os termos "HTLV" e "gravidez", tendo sido localizados um total de 19 artigos.
Após avaliação dos resumos dos artigos por dois revisores, foram excluídos 12 artigos por estarem em duplicata ou não se enquadrarem nos critérios de inclusão e exclusão pré-estabelecidos. Restaram 27 artigos que, após serem lidos na íntegra, foram excluídos mais 15 artigos pelos critérios adotados. Essa seleção resultou em 12 artigos que foram utilizados para formular os resultados. Dentre os 15 estudos excluídos, seis foram reincluídos como fonte de dados para enriquecer a introdução e a discussão. Outros 12 artigos novos também foram utilizados com a mesma finalidade. A pesquisa finalizou com 30 fontes bibliográficas (Quadro 1).

\section{A infecção pelo HTLV-1/2 no Brasil}

O Brasil é um dos países com maior número absoluto de infectados no mundo, com estimativas variando entre 800.000 e 2,5 milhões de pessoas.1,12,13 Esta amplitude nas estimativas deve-se à falta de dados oficiais sobre a doença e à escassez de

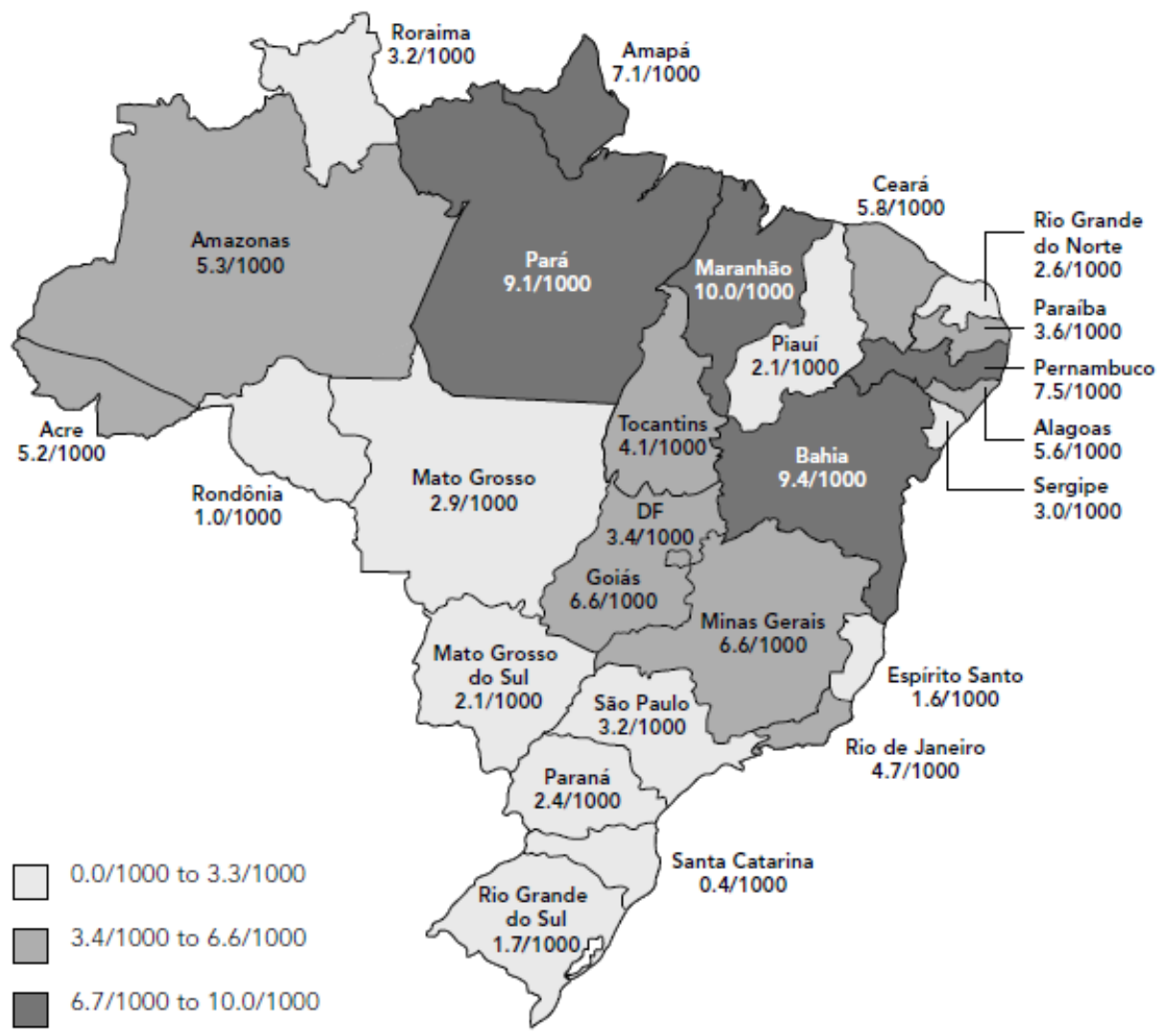

Figura 2. Prevalência de HTLV-1/2 em doadores de sangue rastreados no Brasil entre 1995 e 2000. Reproduzido com permissão dos autores. ${ }^{18}$ 
trabalhos avaliando a prevalência da infecção na população em geral. O único grande estudo nacional que abordou uma população não selecionada foi realizado em Salvador (BA) e encontrou a maior prevalência da infecção pelo HTLV-1 no país (1,76\% na população geral, sendo 1,2\% em homens e $2 \%$ em mulheres), estimando-se 40.000 infectados. ${ }^{14}$

Os estudos são realizados mais frequentemente entre doadores de sangue e populações selecionadas como usuários de drogas injetáveis e pacientes com outras doenças sexualmente transmissíveis. Deste modo, informações sobre a infecção por HTLV-1/2 em gestantes brasileiras ainda são relativamente recentes e pontuais. Os dados já publicados sobre o tema evidenciam importantes variações regionais na prevalência, indo de 0,13\% no Mato Grosso do Sul a 0,8\% na Bahia..15,16 Apesar de ainda não haver dados publicados sobre a prevalência da infecção em nosso estado, o projeto
"Estudo da prevalência e da transmissão vertical de HIV, HTLV e Sífilis em gestantes da Região Metropolitana do Rio de Janeiro" - desenvolvido por um grupo de pesquisadores da UERJ e aceito para publicação no periódico PLOS Neglected Tropical Diseases - identificou prevalência de 0,66\% em 1204 parturientes na região metropolitana do Rio de Janeiro. O teste de triagem durante a gestação não é preconizado como rotina no Estado do Rio de Janeiro, mas, tendo em vista a prevalência encontrada, que pode corresponder à segunda maior do Brasil, os coordenadores do projeto propuseram a inclusão do exame para o HTLV na rotina pré-natal do HUPE/UERJ.

Sabe-se que as gestantes representam melhor a população em geral do que os doadores de sangue, porém ambos refletem de alguma forma a endemicidade da infecção. Em um estudo envolvendo diversos países europeus, foi demonstrado que a so-

Tabela 1. Estudos da infecção por HTLV-1/2 em gestantes e puérperas no Brasil.

\begin{tabular}{|c|c|c|c|c|c|}
\hline Autor/Ano & Local & n & Prevalência HTLV* (\%) & $\begin{array}{c}\text { Evolução com } \\
\text { aborto (\%) }\end{array}$ & $\begin{array}{c}\text { História } \\
\text { abortos (\%) }\end{array}$ \\
\hline Broutet et al, $1996^{25}$ & Fortaleza, CE & 814 & 0,12 & NA & NA \\
\hline $\begin{array}{l}\text { Bittencourt et al, } \\
2001^{16}\end{array}$ & Salvador, BA & 6.754 & $0,84(0,59-1,03)$ & 3,8 & 9,6 \\
\hline $\begin{array}{l}\text { Olbrich Neto et al, } \\
2004^{26}\end{array}$ & Botucatu, SP & 913 & $0,2(0,04-0,88)$ & NA & NA \\
\hline Oliveira et al, $2006^{27}$ & Goiás, GO & 15.485 & 0,1 & NA & NA \\
\hline $\begin{array}{l}\text { Figueiró-Filho et al, } \\
2007^{28}\end{array}$ & MS & 32.512 & $0,13(0,08-0,16)$ & NA & NA \\
\hline $\begin{array}{l}\text { Dal Fabbro et al, } \\
2008^{15}\end{array}$ & MS & 116.689 & 0,13 & 6,7 & 26,8 \\
\hline $\begin{array}{l}\text { Magalhães et al, } \\
2008^{21}\end{array}$ & Cruz das Almas, BA & 408 & 0,98 & NA & NA \\
\hline Ydi et al, $2009^{29}$ & Cuiabá, MT & 2.965 & $0,2(0,10-0,51)$ & NA & NA \\
\hline Lima et al, $2009^{23}$ & Vitória, ES & 534 & $0,6^{\S}$ & NA & 22,6 \\
\hline Ribeiro et al, $2010^{30}$ & MG & 55.293 & 0,076 & NA & NA \\
\hline Sequeira et al, $2012^{22}$ & Pará & 13.382 & 0.3 & NA & 29,7 \\
\hline $\begin{array}{l}\text { Guimarães de Souza } \\
\text { et al, } 2012^{12}\end{array}$ & São Luís, MA & 2.044 & 0,3 & NA & NA \\
\hline
\end{tabular}

NA = não avaliado; * IC95\%;§ resultados de ELISA não confirmados. 
roprevalência para HTLV é proporcional (em média de 5 a 10 vezes maior) em gestantes e em doadores de sangue na mesma área. ${ }^{17}$ Desta forma, a mesma peculiaridade na distribuição geográfica da infecção pode ser antecipada em ambas as populações.

Uma pesquisa detalhada avaliou a prevalência da infecção por HTLV-1/2 em doadores de sangue das 27 capitais brasileiras. Mais de seis milhões de doações foram avaliadas, entre 1995 e 2000, por meio dos dados de bancos de sangue públicos. A heterogeneidade entre as regiões foi considerável, variando de 0,4/1.000 em Florianópolis (SC) a uma taxa 25 vezes maior (10,0/1.000) em São Luís (MA). É importante salientar o padrão de distribuição da doença, com maior soroprevalência nos estados Norte e Nordeste (Pará, Pernambuco, Bahia e Maranhão) e taxas significativamente mais baixas nos estados do Sul, como Santa Catarina (Figura 2). ${ }^{18}$ Estes achados foram corroborados por uma publicação mais recente em que a prevalência de HTLV-1/2 em doadores de sangue de Pernambuco foi mais que o dobro da de São Paulo (2,22/1.000 x 1,01/1.000). ${ }^{19}$ Infelizmente não há um estudo tão abrangente e metodologicamente criterioso que permita a comparação direta dos dados da infecção em grávidas com os de doadores de sangue obtidos por Catalan-Soares. ${ }^{18}$

A heterogeneidade regional da prevalência de HTLV-1/2 no Brasil pode ser explicada pelas características étnicas da população, pois o Nordeste tem maior proporção de descendentes de africanos e ameríndios do que o Sul. A endemicidade do HTLV-2 foi documentada por estudos epidemiológicos em grupos indígenas da Amazônia. ${ }^{20}$ Por outro lado, evidências filogenéticas demonstraram a introdução das cepas de HTLV-1 na Bahia por meio da migração forçada da África, ${ }^{14,21}$ que é a maior área endêmica de HTLV-1 no mundo, sendo considerada o local de origem dos retrovírus humanos. No entanto, mesmo na África, a soroprevalência para HTLV em gestantes apresenta significativa variabilidade, indo de 0,2\% na África do Sul, passando por 0,7\% na República do Congo e alcançando 5,5\% na Nigéria.'

\section{Impacto da infecção por HTLV-1/2 no ciclo gravídico-puerperal}

Nenhum dos artigos sobre HTLV-1/2 em gestantes e puérperas brasileiras abordou especificamente o impacto da infecção no desfecho da gestação, e apenas quatro fazem referência à história obstétrica, mais especificamente de abortamento espontâneo (Tabela 1).

Os estudos conduzidos no Mato Grosso do Sul, Pará e Espírito Santo, que juntos contabilizam mais de 130.000 pacientes, relatam incidência de abortamento de $26,8 \%, 29,7 \%$ e $22,6 \%$ respectivamente, ${ }^{15,22,23}$ notavelmente maior do que a esperada. Por outro lado, a taxa encontrada por Bittencourt e colaboradores em gestantes de Salvador foi de cerca de $9 \%$, semelhante à da população em geral. ${ }^{16}$ Dal Fabbro e colaboradores foram os únicos a identificarem mulheres com dois abortamentos ou mais, o que ocorreu em $0,8 \%$ dos casos e foi, portanto, proporcional à incidência de abortamentos de repetição (três ou mais) na população. ${ }^{15}$

Não foram encontradas publicações que avaliassem a associação da infecção por HTLV-1/2 a outros desfechos gestacionais desfavoráveis, como alterações placentárias, mortalidade perinatal e baixo peso ao nascer, por exemplo.

O maior impacto da infecção pelo HTLV em mulheres talvez não ocorra durante a gravidez, mas sim no puerpério. No Brasil, a recomendação para as mães HTLV positivo é que não amamentem seus filhos, nem doem leite para Bancos de Leite Humano (BLH), devido ao risco de transmissão do vírus. No entanto, o ato de amamentar contempla não apenas aspectos biológicos, mas também determinantes culturais, comportamentais e valores sociais dominantes de cada época. A imagem da amamentação, de alguma maneira, tem povoado o mundo das mulheres enquanto símbolo representativo da maternidade, construído social e culturalmente ao longo dos tempos, e paradoxalmente tido como determinação biológica da espécie. ${ }^{24}$ Neste sentido, torna-se fundamental compreender que a mulher, ao ser impedida de amamentar, poderá passar por problemas emocionais, sociais e até mesmo financeiros, devido ao alto custo da alimentação artificial.

\section{Comentários finais}

A transmissão do HTLV-1/2 pode ocorrer de três formas: contato sexual, contato com sangue ou secreções e por transmissão vertical (TV). A 
TV ocorre em cerca de $20 \%$ dos filhos de mães infectadas, sendo sua principal via o aleitamento materno prolongado. A prevalência da infecção sofre variações regionais, indo de $0,13 \%$ no Mato Grosso do Sul a 0,8\% na Bahia. O maior impacto da infecção pelo HTLV-1/2 em mulheres ocorre no puerpério, em função da recomendação para a não prática do aleitamento materno.

\section{Referências}

1. Gessain A, Cassar O. Epidemiological aspects and world distribution of HTLV-1 infection. Front Microbiol. 2012;3:388. http://dx.doi.org/10.3389/ fmicb.2012.00388

2. Gonçalves DU, Proietti FA, Ribas JGR, Araújo MG, Pinheiro SR, Guedes AC, et al. Epidemiology, Treatment, and Prevention of Human T-Cell Leukemia Virus Type I-Associated Diseases. Clin Microbiol Rev. 2010;23(3):577-89. http://dx.doi. org/10.1128/CMR.00063-09

3. Hino S. Establishment of the Milk-borne transmission as a key factor for the peculiar endemicity of human T-lymphotropic virus type I (HTLV-1): the ATL Prevention Program Nagasaki. Proc Jpn Acad Ser B Phys Biol Sci. 2011;87(4):152-66.

4. Takahashi K, Takezaki T, Oki T, Kawakami K, Yashiki S, Fujiyoshi T, et al. Inhibitory effect of maternal antibody on Mother-to-Child transmission of human T-lymphotropic virus type I: the Motherto-Child Transmission Study Group. Int J Cancer. 1991;49(5):673-7. http://dx.doi.org/10.1002/ ijc. 2910490508

5. Katamine S, Moriuchi R, Yamamoto T, Terada K, Eguchi K, Tsuji Y, et al. HTLV-1 proviral DNA in umbilical cord blood of babies born to carrier mothers. Lancet. 1994;343(8909):1326-7.

6. Ureta-Vidal A, Angelin-Duclos C, Tortevoye P, Murphy E, Lepère JF, Buigues RP, et al. Motherto-child transmission of human T-cell-leukemia/ lymphoma virus type I: implication of high antiviral antibody titer and high proviral load in carrier mothers. Int J Cancer. 1999;82(6):832-6.

7. Fujino T, Iwamoto I, Otsuka H, Ikeda T, Takesako S, Nagata Y. Apoptosis in placentas from human T-lymphotropic virus type I-seropositive pregnant women: a possible defense mechanism against transmission from mother to fetus. Obstet Gynecol. 1999;94(2):279-83.

8. Fujino T, Nagata Y. HTLV-1 transmission from mother to child. J Reprod Immunol. 2000;47(2):197-206.

9. Fujino T, Fujiyoshi T, Yashiki S, Sonoda S, Otsuka H, Nagata Y. HTLV-1 transmission from mother to fetus via placenta. Lancet. 1992;340(8828):1157.
10. Saito S, Furuki K, Ando Y, Tanigawa T, Kakimoto K, Moriyama I, et al. Identification of HTLV-1 sequence in cord blood mononuclear cells of neonates born to HTLV-1 antigen/antibody-positive mothers by polymerase chain reaction. Jpn J Cancer Res. 1990;81(9):890-5.

11. Satow $\mathrm{Y}$, Hashido M, Ishikawa K, Honda H, Mizuno M, Kawana T, et al. Detection of HTLV-1 antigen in peripheral and cord blood lymphocytes from carrier mothers. Lancet. 1991;338(8772):915-6.

12. Souza VG, Martins ML, Carneiro-Proietti AB, Januário JN, Ladeira RV, Silva CM, et al. High prevalence of HTLV-1 and 2 viruses in pregnant women in São Luis, state of Maranhão, Brazil. ver Soc Bras Med Trop. 2012;45(2):159-62.

13. Carneiro-Proietti AB, Ribas JG, Catalan-Soares BC, Martins ML, Brito-Melo GE, Martins-Filho OA, et al. Infecção e doença pelos vírus linfotrópicos humanos de célula T (HTLV-1/2) no Brasil. ver Soc Bras Med Trop. 2002;35:499-508.

14. Dourado I, Alcantara LC, Barreto ML, Teixeira MG, Galvão-Castro B. HTLV-1 in the general population of Salvador, Brazil: a city with African ethnic and sociodemographic characteristics. J Acquir Immune Defic Syndr. 2003;34(5):527-31.

15. Dal Fabbro MMFJ, Cunha RV, Bóia MN, Portela P, Botelho CA, Freitas GMBF, et al. HTLV 1/2 infection: prenatal performance as a disease control strategy in State of Mato Grosso do Sul. Rev Soc Bras Med Trop. 2008;41(2):148-51.

16. Bittencourt AL, Dourado I, Filho PB, Santos M, Valadão E, Alcantara LC, et al. Human T-cell lymphotropic virus type I infection among pregnant women in northeastern Brazil. J Acquir Immune Defic Syndr. 2001;26(5):490-4

17. Taylor GP, Bodeus M, Courtois F, Pauli G, del Mistro A, Machuca A, et al. The Seroepidemiology of Human T-Lymphotropic Viruses Types I and II in Europe: A Prospective Study in Pregnant Women. J Acquir Immune Defic Syndr 2005;38(1):104-109.

18. Catalan-Soares B, Carneiro-Proietti AB, Proietti FA. Interdisciplinary HTLV Research Group. Heterogeneous geographic distribution of human T-cell lymphotropic viruses I and II (HTLV-1/2): serological screening prevalence rates in blood donors from large urban areas in Brazil. Cad Saude Publica. 2005;21(3):926-31.

19. Carneiro-Proietti AB, Sabino EC, Leão S, Salles NA, Loureiro P, Sarr M, et al. Human T-lymphotropic virus type I and type II seroprevalence, incidence, and residual transfusion risk among blood donors in Brazil during 2007-2009. AIDS Res Hum Retroviruses. 2012;28(10):1265-72.

20. Ishak R, Vallinoto AC, Azevedo VN, Ishak MOG. Epidemiological aspects of retrovirus (HTLV) infection among Indian populations in the Amazon Region of 
Brazil. Cad Saúde Pública. 2003;19(4):901-14.

21. Magalhães T, Mota-Miranda AC, Alcantara LC, Olavarria V, Galvão-Castro B, Rios-Grassi MF. Phylogenetic and molecular analysis of HTLV-1 isolates from a medium sized town in northern of Brazil: tracing a common origin of the virus from the most endemic city in the country. J Med Virol. 2008;80(11):2040-5.

22. Sequeira CG, Tamegão-Lopes BP, Santos EJM, Ventura AMR, Moraes-Pinto MI, Succi RCM. Descriptive study of HTLV infection in a population of pregnant women from the state of Pará, Northern Brazil / Estudo descritivo da infecção pelo HTLV em uma população de gestantes do Estado do Pará, norte do Brasil. Rev Soc Bras Med Trop. 2012;45(4):453-6.

23. Lima LH, Viana MC. Prevalence and risk factors for HIV, syphilis, hepatitis B, hepatitis C, and HTLV-1/2 infection in low-income postpartum and pregnant women in Greater Metropolitan Vitória, Espírito Santo State, Brazil. Cad Saúde Pública. 2009;25(3):668-76.

24. Silva IA. Psicologia, estresse e amamentação. In: Santos LA Jr, editor. A mama no ciclo gravídicopuerperal. São Paulo: Atheneu; 2000. p. 67.

25. Broutet N, Sousa AQ, Basilio FP, Sá HL, Simon F, Dabis F. Prevalence of HIV-1, HIV-2 and HTLV antibody in Fortaleza, Ceará, Brazil, 1993-1994. Int J STD AIDS. 1996;7(5):365-9.

26. OlbrichNeto J, Meira DA.Soroprevalence of HTLV$1 / 2$, HIV, siphylis and toxoplasmosis among pregnant women seen at Botucatu - São Paulo - Brazil: risk factors for HTLV-1/2 infection. Rev Soc Bras Med Trop. 2004;37(1):28-32

27. Oliveira SR, Avelino MM. Human T-cell lymphotropic virus type I seroprevalence among pregnant women in Goiânia, GO, Brazil. Rev Bras Ginecol Obstet. 2006;28(8):467-72

28. Figueiró-Filho EA, Senefonte FR, Lopes AH, de Morais OO, Souza Júnior VG, Maia TL, et al. Frequency of HIV-1, rubella, syphilis, toxoplasmosis, cytomegalovirus, simple herpes virus, hepatitis B, hepatitis C, Chagas disease and HTLV I/II infection in pregnant women of State of Mato Grosso do Sul. Rev Soc Bras Med Trop. 2007;40(2):181-7.

29. Ydy RR, Ferreira D, Souto FJ, Fontes CJ. Prevalence of human T-cell lymphotropic virus (HTLV-1/2) infection among puerperae in Cuiabá, Mato Grosso, 2006. Rev Soc Bras Med Trop. 2009;42(1):28-32.
30. Ribeiro M, Proietti F, Martins M, Januário J, Puglia Ladeira R, Oliveira M, et al. Geographic distribution of human T-lymphotropic virus types 1 and 2 among mothers of newborns tested during neonatal screening, Minas Gerais, Brazil. Rev Panam Salud Publica. 2010;27(5):330-7.

\section{Danielle B. S. Barmpas}

Programa de Pós-graduação em Ciências Médicas. Faculdade de Ciências Médicas. Universidade do Estado do Rio de Janeiro. Rio de Janeiro, RJ, Brasil.

\section{Denise L. M. Monteiro}

Disciplina de Obstetrícia. Departamento de Ginecologia e Obstetrícia. Centro Universitário Serra dos Órgãos (UNIFESO). Teresópolis, RJ, Brasil.

Disciplina de Obstetrícia. Departamento de Ginecologia e Obstetrícia. Faculdade de Ciências Médicas da Universidade do Estado do Rio de Janeiro. Rio de Janeiro, RJ, Brasil.

\section{Stella R. Taquette}

Disciplina de Medicina de Adolescentes. Departamento de Medicina Interna. Faculdade de Ciências Médicas. Universidade do Estado do Rio de Janeiro. Rio de Janeiro, RJ, Brasil.

\section{Alexandre J. B. Trajano}

Departamento de Ginecologia e Obstetrícia. Faculdade de Ciências Médicas. Universidade do Estado do Rio de Janeiro. Rio de Janeiro, RJ, Brasil.

Departamento de Ginecologia e Obstetrícia. Faculdade de Medicina. Universidade Unigranrio. Rio de Janeiro, RJ, Brasil.

\section{Roberta M. Raupp}

Programa de Pós-graduação em Informação e Comunicação em Saúde. Instituto de Comunicação e Informação Científica e Tecnológica em Saúde. Fiocruz. Rio de Janeiro, RJ, Brasil.

\section{Fátima R. D. Miranda}

Disciplina de Obstetrícia. Departamento Ginecologia e Obstetrícia. Faculdade de Ciências Médicas. Universidade do Estado do Rio de Janeiro. Rio de Janeiro, RJ, Brasil.

\section{Nadia C. P. Rodrigues}

Disciplina de Epidemiologia e Bioestatística. Departamento de Tecnologias da Informação e Educação em Saúde. Faculdade de Ciências Médicas. Universidade do Estado do Rio de Janeiro. Rio de Janeiro, RJ, Brasil. 